\title{
"Enfermedad de las tres mentiras": síndrome de la úlcera rectal solitaria
}

\author{
L. Crespo Pérez, V. Moreira Vicente, C. Redondo Verge ${ }^{1}$, A. López San Román y J. M. Milicua Salamero \\ Departamento de Gastroenterología. ${ }^{~}$ Departamento de Anatomía Patológica. Hospital Universitario Ramón y Cajal. \\ Madrid
}

\section{RESUMEN}

El síndrome de la úlcera rectal solitaria es una entidad benigna poco común cuyos síntomas más frecuentes son la rectorragia y el dolor anal. Los hallazgos anatomopatológicos son típicos. Se suele evidenciar engrosamiento de la mucosa, elongación y distorsión de las glándulas, una lámina propia edematosa con gran cantidad de colágena y engrosamiento de la muscularis mucosae. El diagnóstico se realiza mediante endoscopia con toma de biopsias. No siempre se trata de lesiones ulceradas. Se suele localizar preferentemente en la cara rectal anterior y/o lateral, aunque hasta un $30 \%$ de las lesiones son múltiples, existiendo casos de afectación del colon sigmoide y descendente. Por tanto, la presentación puede ser heterogénea y este es el motivo por el que esta entidad es también conocida como la "enfermedad de las tres mentiras". Presentamos un caso de síndrome de la ulcera rectal solitaria manifestado endoscópicamente como una placa eritematosa localizada en la cara lateral izquierda del recto.

Palabras clave: Síndrome de la úlcera rectal solitaria. Hemorragia digestive baja. Molestias anales.

\begin{abstract}
Solitary rectal ulcer syndrome is an uncommon benign condition characterized by rectal bleeding, passage of mucus, and pain. Histological features are well established as obliteration of the lamina propria by fibrosis and smooth-muscle fibers extending from a thickened muscularis mucosa to the lumen. Diagnosis can usually be made on sigmoidoscopy, and biopsies should always be taken. Ulceration is not universally present, and polypoid, non-ulcerated lesions and erythematous areas are also seen. The lesion or lesions are most often found on the anterior or anterolateral wall of the rectum, although they can also be located in the left colon and be more extensive or even circumferential. Lesions are multiple in 30 percent of cases. These are the reasons why this entity is also known as "the disease of three lies". We report a case of solitary rectal ulcer syndrome presenting at endoscopy with an erythematous area on the left side wall of the rectum.
\end{abstract}

Key words: Solitary rectal ulcer syndrome. Lower digestive hemorraghe. Anal discomfort.

Crespo Pérez L, Moreira Vicente V, Redondo Verge C, López San Román A, Milicua Salamero JM. "Enfermedad de las tres mentiras": síndrome de la úlcera rectal solitaria. Rev Esp Enferm Dig 2007; 99: 663-666.

\section{INTRODUCCIÓN}

El síndrome de la úlcera rectal solitaria (SURS) es un trastorno crónico benigno caracterizado por una combinación de síntomas, hallazgos endoscópicos y alteraciones histopatológicas $(1,2)$. En 1969, Madigan y Morson establecieron por primera vez los criterios clínicos e histológicos para definir esta entidad basándose en una serie de 68 casos (3). Se trata de una entidad poco común cuya incidencia se estima en 1-3 casos por 100.000 habitantes y año $(4,5)$.

\section{Recibido: 18-04-07.}

Aceptado: 11-05-07.

Correspondencia: Laura Crespo Pérez. Departamento de Gastroenterología. Hospital Universitario Ramón y Cajal. Ctra. de Colmenar, km 9,100 28034 Madrid.Fax: 9133687 85.e-mail: lcreper@yahoo.es
Como consecuencia directa de su rareza esta patología inicialmente se puede confundir con otras enfermedades digestivas, especialmente con neoplasias o la enfermedad inflamatoria intestinal. En una serie de 25 pacientes con SURS y diagnóstico incorrecto inicial, el tiempo medio desde el momento de la consulta hasta el diagnóstico definitivo fue 5 años; los pacientes con lesiones ulceradas o con "parches" de mucosa hiperémica habían sido etiquetados de colitis ulcerosa o enfermedad de Crohn, y las formas polipoides habían sido confundidas con pólipos neoplásicos (6).

Presentamos un caso de SURS manifestado endoscópicamente como una placa eritematosa localizada en la cara lateral izquierda del recto. Las biopsias confirmaron el diagnóstico de SURS, aunque el aspecto macroscópico inicialmente sugirió una enfermedad inflamatoria intestinal o una úlcera estercorácea. 


\section{CASO CLÍNICO}

Mujer de 42 años estudiada en Consultas de Gastroenterología desde enero de 2005 por presentar rectorragia escasa al final de la deposición junto con tenesmo y dolor anal de dos años de evolución. La paciente no tenía ningún antecedente familiar ni personal de interés ni seguía ningún tratamiento médico de forma habitual. En la exploración física tan sólo se objetivó en el tacto rectal una zona indurada en la pared lateral izquierda del recto. Se realizó una analítica completa que resultó rigurosamente normal. Ante este hallazgo se decidió realizar una colonoscopia encontrándose dos pólipos milimétricos en ampolla rectal, hemorroides internas (una de ellas prolapsada) y en la cara lateral izquierda de la ampolla rectal un área eritematosa y levemente sobreelevada, de aproximadamente $2 \times 2 \mathrm{~cm}$, de bordes geográficos y con un aspecto indeterminado de la que se tomaron biopsias. Se planteó inicialmente el diagnóstico diferencial entre enfermedad inflamatoria intestinal (colitis ulcerosa), úlcera estercorárea y síndrome de la úlcera rectal solitaria. La anatomía patológica demostró un engrosamiento de la mucosa, con elongación y distorsión de las glándulas y una lámina propia edematosa con gran cantidad de colágeno y fibroblastos, todo ello compatible con el diagnóstico de síndrome de la úlcera rectal solitaria (Figs. 1 y 2). Se realizó una manometría anorrectal que evidenció un volumen rectal máximo tolerable discretamente reducido y un tiempo de expulsión del balón mínimamente alargado.

\section{RESULTADOS}

Se inició entonces tratamiento simultáneo con fibra oral, enemas de $2 \mathrm{~g}$ de sucralfato cada $12 \mathrm{~h}$ durante 3 meses y sesiones de biofeedback durante 9 meses. La paciente mejoró notablemente, desapareciendo las molestias anales y disminuyendo la cuantía de la rectorragia al segundo mes, encontrándose totalmente asintomática al finalizar el tratamiento. Se realizó una nueva colonoscopia en octubre de 2005 objetivándose en la mucosa del recto distal una zona muy circunscrita de mucosa edematosa e intensamente eritematosa de la que de nuevo se tomaron biopsias que confirmaron el diagnóstico previo. La paciente actualmente (febrero 2007) permanece asintomática aunque los hallazgos endoscópicos persisten.

\section{DISCUSIÓN}

El síndrome de la úlcera rectal solitaria (SURS) es un trastorno crónico benigno cuyo diagnóstico se fundamenta en la clínica, la apariencia endoscópica y fundamentalmente en los hallazgos histológicos (1). El síntoma más común es la rectorragia (60\%), habitualmente en forma de sangre roja en pequeña cuantía mezclada con la deposición. Otro síntoma frecuente es la eliminación rec-

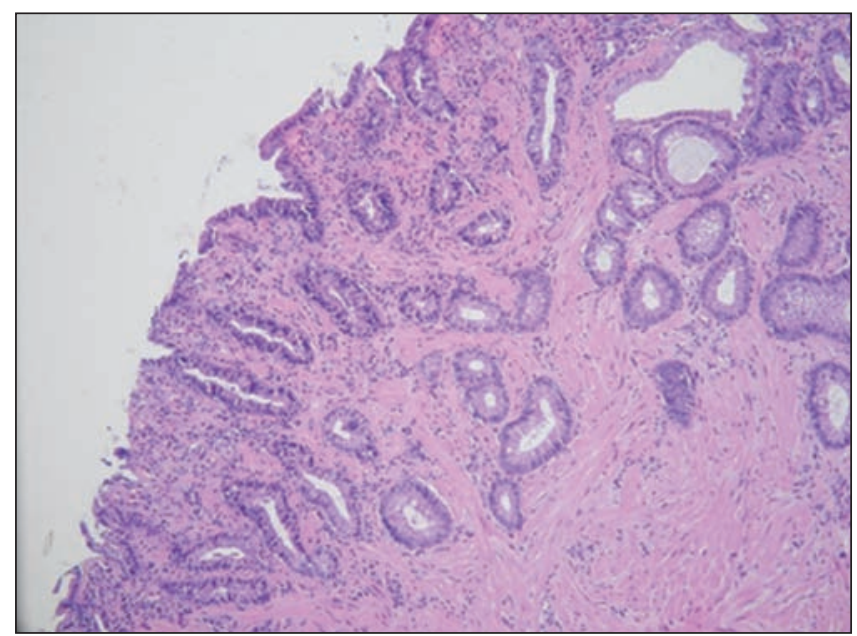

Fig. 1. A pequeño aumento se observa obliteración fibromuscular de la lámina propia.

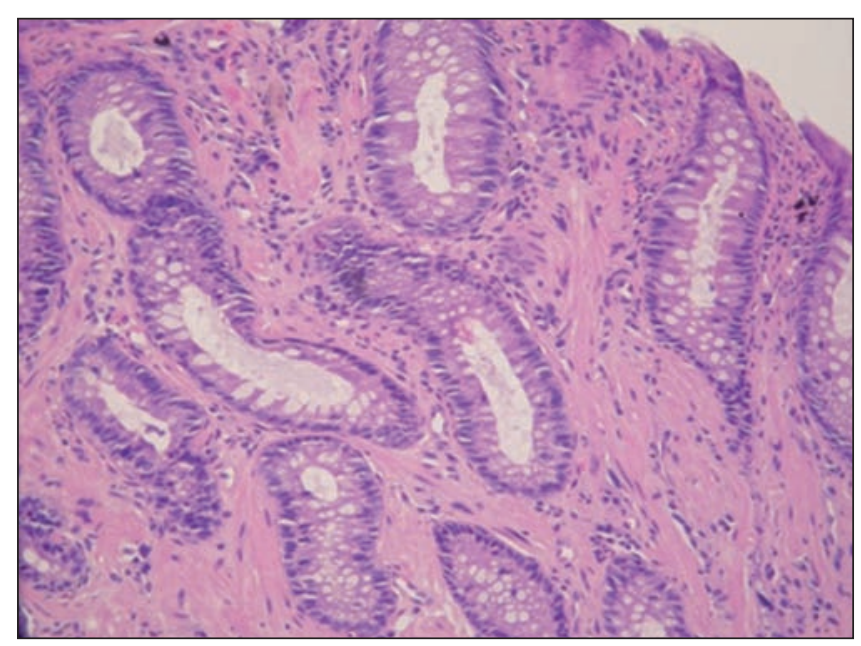

Fig. 2. A mayor aumento se aprecia verticalización de las fibras musculares lisas que ascienden a la superficie de la mucosa.

tal de moco (18\%). El estreñimiento está presente hasta en el 55\% de los casos y suele ser de tipo obstructivo distal caracterizado por un esfuerzo excesivo al defecar, con deseo permanente de defecar y a veces con sensación de "taponamiento" debido al prolapso mucoso de la pared rectal anterior. En ocasiones se asocia con dolor abdominal hipogástrico, lumbosacro o anorrectal. Se estima que hasta un $20 \%$ de los pacientes tienen diarrea en lugar de estreñimiento y sorprendentemente hasta el $25 \%$ de los pacientes no presenta ningún síntoma, realizándose el diagnóstico de forma incidental (7). El aspecto macroscópico típico, presente en el 57\% de los casos, es el de una úlcera entre 0,5 y $5 \mathrm{~cm}$, superficial, rodeada ocasionalmente por un halo eritematoso, de fondo blanquecino, gris o amarillento. La morfología de la úlcera es variable, pudiendo ser redondeada, ovalada, lineal, serpinginosa o estrellada. Suelen ser lesiones únicas localizadas en la cara anterior y/o lateral del recto. De todos modos, hasta un $30 \%$ de las lesiones son múltiples y existen casos des- 
critos de afectación del colon sigmoide y descendente (8). En el $25 \%$ de los casos se identifican lesiones polipoides y sólo en un 18\% "parches" de mucosa hiperémica. El caso que exponemos se manifestó endoscópicamente como una zona eritematosa con bordes bien definidos, la variante menos común del SURS. Por tanto, la presentación de esta patología puede ser muy heterogénea, ya que con frecuencia no existe una imagen clara de úlcera o se aprecian lesiones múltiples o las lesiones no están localizadas en el recto, motivo por el cual se le ha denominado "enfermedad de las tres mentiras". La toma de biopsias es obligada para poder establecer el diagnóstico definitivo de forma segura. En ocasiones estas lesiones son subestimadas durante la exploración endoscópica y no son biopsiadas, especialmente la variante de nuestro caso clínico, al ser simples "parches" de mucosa edematosa y eritematosa que pueden confundirse con pequeños traumatismos del endoscopio sobre la mucosa colorrectal o con lesiones mucosas secundarias a la limpieza del colon (más frecuentemente con preparados derivados de los fosfatos). Los hallazgos anatomopatológicos son típicos y han sido bien documentados (Figs. 1 y 2). En general se suele evidenciar engrosamiento de la mucosa, elongación y distorsión de las glándulas, una lámina propia edematosa con gran cantidad de colágena y con variable proliferación fibromuscular ("obliteración fibromuscular") y engrosamiento de la muscularis mисоsae, con fibras musculares que ascienden verticalizadas y engloban las criptas produciendo desorganización de la arquitectura (8). La manometría anorrectal tiene un papel escaso en el diagnóstico aunque importante, ya que permite investigar la integridad neuromuscular del sistema esfinteriano. La entidad con la que más seriamente hay que plantearse el diagnóstico diferencial es la neoplasia colorrectal. Clásicamente se considera que el SURS es una patología crónica y benigna. Tsuschida y cols. describieron un caso de SURS con un foco de adenocarcinoma bien diferenciado invadiendo la submucosa en el centro de la úlcera rectal y glándulas displásicas adyacentes al foco de adenocarcinoma sugiriéndose así la posibilidad de transformación maligna del SURS (9). Ocasionalmente el SURS resulta difícil de diferenciar de la enfermedad inflamatoria intestinal, especialmente en casos de colitis ulcerosa. Uza y cols. describieron recientemente el caso de un paciente con pancolitis ulcerosa de larga de evolución, al que se realizó una colonoscopia objetivándose la existencia de una masa en la cara rectal anterior con una úlcera lineal en su superficie. Los resultados anatomopatológicos fueron concluyentes ya que se demostró una obliteración fibromuscular de la lámina propia, característico del SURS. Se realizaron colonoscopias anuales con toma de biopsias, sin haberse demostrado áreas de displasia en ninguna ocasión (10). Otras entidades con las que hay que establecer diagnóstico diferencial son las colitis infecciosas, la isquémica, la colitis cística profunda, la úlcera estercorácea y las lesiones rectales secundarias a traumatismos digitales. La causa y la verdadera fisiopatología del SURS son desconocidas y probablemente su origen es multifactorial. La contracción persistente del músculo puborrectal durante la defecación hace persistir un ángulo anorrectal agudo que mantiene cerrado el conducto anal, lo que desencadena un aumento de la presión intrarrectal en los pacientes con SURS (11). Este esfuerzo propulsa la mucosa de la pared rectal anterior contra el conducto anal y el prolapso repetido de la mucosa ocasionaría isquemia y finalmente su ulceración. El traumatismo directo por los intentos de eliminar de forma digital heces duras del recto también se ha planteado como causa de este síndrome (12). Por último ha podido documentarse una etiología infecciosa. Así, se ha descrito un caso de SURS atípico asociado a infección por Mycobacterium chelonae (13). El tratamiento del SURS es problemático y no se pueden realizar recomendaciones terapéuticas firmes. Conviene tranquilizar al paciente en cuanto a la naturaleza benigna de la enfermedad y solicitar su colaboración durante un largo periodo de tiempo. Existen cuatro pilares básicos de tratamiento: médidas higiénico-dietéticas, tratamiento farmacológico, "biofeedback" y cirugía. De todos modos, es frecuente que el paciente presente mejoría sintomática tras la utilización de las distintas posibilidades terapéuticas, si bien los hallazgos endoscópicos y/o histológicos pueden no desaparecer (tal y como ocurrió en el caso que presentamos). Nuestra paciente fue tratada con fibra y enemas de sucralfato asociados a biofeedback, documentándose mejoría clínica si bien los hallazgos endoscópicos persistieron. La primera medida dietética será el incremento de la ingesta de fibra dietética (ingesta de vegetales, frutas, laxantes formadores de masa o mucílagos). La respuesta es variable, siendo los pacientes con prolapso rectal asociado los que menos se beneficiarán de estas medidas. En general son muy pocos los fármacos empleados en el manejo del SURS, algunos de ellos los mismos que se emplean en el tratamiento de la enfermedad inflamatoria intestinal. Zagar y cols. realizaron un estudio abierto en el que los pacientes recibían enemas con $2 \mathrm{~g}$ de sucralfato dos veces al día durante 6 semanas, con una media de seguimiento de 8 meses. Se realizaba una exploración endoscópica antes y después del tratamiento, demostrándose mejoría macroscópica en todos los pacientes (14). Los esteroides tópicos y los aminosalicilatos no resultan útiles (15). Los alentadores resultados obtenidos por las terapias conductuales en el estreñimiento han animado a diversos autores a aplicarlas en el tratamiento del SURS y así el "biofeedback" se ha utilizado como terapia única y como adyuvante a la cirugía se trata de una técnica no invasiva y libre de efectos adversos que intenta incidir tanto en aspectos psicológicos como en aspectos fisiológicos (normalizar la coordinación de los músculos del suelo pélvico durante la defecación) (16). Se estima que aproximadamente una tercera parte de los pacientes no responden a las medidas descritas previamente y presentan síntomas incapacitantes que inducen a valorar la indicación de tratamiento 
quirúrgico. De estos pacientes aproximadamente el 50\% tienen un prolapso rectal asociado. La técnica más frecuentemente empleada en estos casos es la resección de la mucosa engrosada y la rectopexia (17).

\section{BIBLIOGRAFÍA}

1. Erdozain JC, Bárcena R, Pérez F, López San Román A, Sánchez J, Gil Grande L. Úlcera solitaria del recto. Rev Esp Enferm Dig 1989; 76: 269-71.

2. Del Val Antoñana A, Moreno-Osset E. Úlcera solitaria de recto, ¿inflamación, infección, isquemia o trastorno motor? Gastroenterología Hepatología 2003; 26: 376-80.

3. Madigan MR, Morson BC. Solitary rectal ulcer of the rectum. Gut 1969; 10: 871-81.

4. Martin CJ, Parks TG, Biggart JD. Solitary rectal ulcer syndrome in Northern Ireland. 1971-1980. Br J Surg 1981; 68: 744-77.

5. Haray PN, Morris-Stiff GJ, Foster ME. Solitary rectal ulcer syndrome: an underdiagnosed condition. Int J Colorect Dis 1997; 12: 313-5.

6. Tjandra JJ; Fazio VW, Petras RE, Lavery IC, Oakley JR, Milson JW Clinical and pathological factors associated with delayed diagnosis in solitary rectal ulcer syndrome. Dis Colon Rectum 1993; 36: 146-53.

7. Tjandra JJ, Fazio VW, Church JM. Clinical conundrum of solitary rectal ulcer. Dis Colon Rectum 1992; 35: 227-34.
8. Sharara AI, Azar C, Amr SS, Haddad M, Eloubeidi MA. Solitary rectal ulcer syndrome: endoscopic spectrum and review of literature. Gastrointest Endosc 2005; 62: 755-62.

9. Tsuschida K, Okayama N, Miyama N. Solitary rectal ulcer syndrome accompanied by submucosal invasive carcinoma. Am J Gastroenterol 1998; 93: 2235-8.

10. Uza N, Nakase H, Nishimura K, Yoshida S, Kawabata K, Chica T. Solitary rectal ulcer syndrome associated with ulcerative colitis. Gastrointest Endosc 2006; 63: 355-6.

11. Wonack NR, Williams NS, Holmfield JHM, Morrison JFB. Pressure and prolapse: the cause of solitary rectal ulceration. Gut 1987; 28 : 1228-33.

12. Contractor TQ, Contractor QQ. Traumatic solitary rectal ulcer in Saudi Arabia. A distinct entity? J Clin Gastroenterol 1995; 21: 298-300.

13. Rodríguez JC, Reyes DM, Royo G, Andrada E, Sillero C. Mycobacterium chelonae y úlcera rectal solitaria. Gastroenterología Hepatología 2000; 23: 474-6.

14. Zagar SA, Khuroo MS, Mahajan R. Sucralfate retention enemas in solitary rectal ulcer. Dis Colon Rectum 1991; 34: 455-7.

15. Flet-Bersma RFJ, Cuesta MA. Rectal prolapse, rectal intussusception, rectocele, and solitary rectal ulcer syndrome. Gastroenterol Clin North Am 2001; 30: 199-222.

16. Rao SSC, Ozturk R, De Ocampo S, Stessman M. Pathophysiology and role of biofeedback therapy in solitary rectal ulcer syndrome. Am J Gastroenterol 2006; 101: 613-8.

17. Sitzler PJ, Kamm MA, Nicholls RJ. Long-term clinical outcome of surgery for solitary rectal ulcer syndrome. Br J Surg 1998: 85: 124650. 